\title{
Monitoring Mine Seismicity in Canada
}

\author{
J. Alexander, C-I. Trifu ESG Canada Inc.
}

First-arrival analogue seismic systems have been employed in Canadian mines since the 1970s. They were automatic, simple and robust, but could not provide post-event confirmation of first arrival times, affecting event location reliability. Moreover, these systems suffered from limited dynamic range, signal loss, and electrical interference. Full waveform event based digital seismic systems were developed toward the end of the 1980s. They offered better dynamic range (12-bit resolution) and better signal quality. The dynamic range has been further increased by the use of 16-bit resolution analogue-to-digital converters throughout the 1990s. Tens of seismic arrays are currently in operation, monitoring volumes on the order of several hundreds of meters to a couple kilometres across with location accuracies ranging from meters to a few tens of meters. Mechanical vibrations from drilling, fans, ore passes and large equipment cause many problems with trigger based systems and can result in data loss. The new generation 24-bit resolution system offers superior dynamic range, exceptional signal quality, and no data loss due to continuous recordings from every sensor in the network. The bottleneck to continuous digital seismic recording has mainly been limitations in telemetry technology, getting the data from each sensor back to a central recording system. New technological advances that now power high-speed Internet communication through standard telephone cable can be used to record continuous digital microseismic data in an industrial environment at sampling frequencies of up to $10 \mathrm{kHz}$.

\section{INTRODUCTION}

\subsection{First-arrival Seismic Systems}

The history of mine seismicity is tied to rockburst type events resulting in damage to underground excavations. The first rockburst in Canada was reported in 1928 and by the 1930s they were recognized as a significant problem. By the 1940s, mines from both Sudbury and Kirkland Lake regions were emerging as hotspots for seismic activity. At Frood mine in Sudbury a lot of smaller magnitude events (microseismicity) located close to mine workings were generated. This was related to the mine design and extraction of the cut and fill stopes. By contrast, Lakeshore mine in Kirkland Lake was generating large magnitude seismic activity related to tectonic stresses and major faults. The mine was closed following a magnitude 4.2 event, which destroyed the lower portion of their $8,000 \mathrm{ft}$ shaft. By the late 1970s and early 1980s a large increase in seismic activity in many deep Canadian mines was resulting in fatalities and mine closures.

The Canadian Rockburst Research Program was created to coordinate the efforts of the mining industry to implement plans for monitoring and analyzing seismicity in mines (Hedley, 1992). During that period, first-arrival based systems were installed (Figure 1). These machines, originally developed and used in Coeur d'Alene district (Idaho), would calculate a rudimentary location and output a stream of first arrival time numbers to a Teletype printer (Blake, 1982).

An interface was developed to expensive mainframe type computers where more elaborate routines would further reduce the data. These systems were robust machines and some are still in operation today. However, the arrival times are not always accurate, without any possibility to identify noise or mispicks, and there is no accurate calculation of event magnitude.

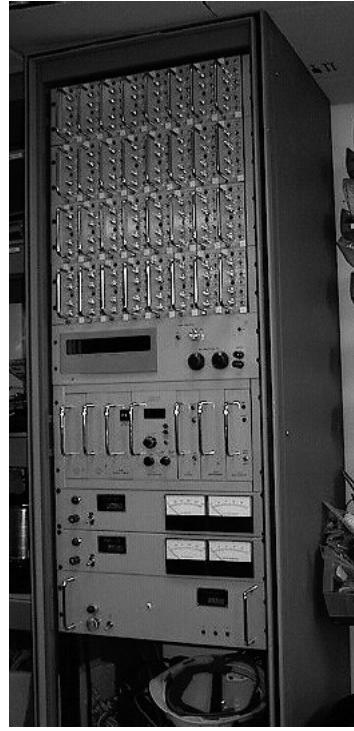

FIG. 1 MP250 first-arrival seismic system from Electrolab (Spokane, WA)

\subsection{Full Waveform Seismic Systems}

At the end of 1980s, full waveform recording systems were developed to overcome the limitations of the firstarrival based systems. Initially, these systems were used in a piggyback configuration but eventually evolved into stand-alone seismic recording systems. They offered the advantage of more accurate first arrival times with the ability to manually check and correct mispicks. This meant more accurate event locations, as well as information about the source of the event including event magnitudes. The advent of personal computers really made these systems widely affordable. They were based on a centralized recording 
system with all sensors powered from one location. Analogue sensor signals were digitized at $20 \mathrm{kHz}$ sampling and 12-bit resolution, limiting the dynamic range to about $70 \mathrm{~dB}$. Signals from all sensors were recorded when a microseismic event triggered the system. Computer programs processed the data automatically picking the first arrival times and calculating the event location and magnitude.

The goal of this study is to provide a review and update of the state of microseismic monitoring in Canada. It includes a presentation of the available technology, the advantages and limitations of various options, as well as examples of applications at several mine sites. The study outlines that the present trend and future direction of development meet the demands of the Canadian mining industry.

\section{CONTEMPORARY SYSTEM CONFIGURATIONS}

Seismic systems in Canadian mines were originally developed to monitor relatively small 3D type ore bodies of several hundred metres to a couple kilometres across, located in hard rock mines at depths below $600-700 \mathrm{~m}$. Typically, these mines generated few of the large earthquake type events commonly experienced with large tabular ore bodies. In order to effectively sample the rockmass, the emphasis was put on measuring smaller microseismic events and determining their position with a high degree of accuracy, usually better than 10m (Trifu and Urbancic, 1997).

\subsection{Response of Geophones vs Accelerometers}

The signal amplitude measured on the seismic system is directly related to the energy released (size of the event), the source-sensor distance and the sensitivity of the sensor. Seismic monitoring systems use either geophone or accelerometer transducers to convert ground vibrations into velocity or acceleration, respectively. The selection of a sensor proportional to velocity or acceleration depends upon the frequencies of interest and the signal levels involved. Figure 2 shows the geophone response with respect to the accelerometer response over the frequency range from 2 to $4,000 \mathrm{~Hz}$.

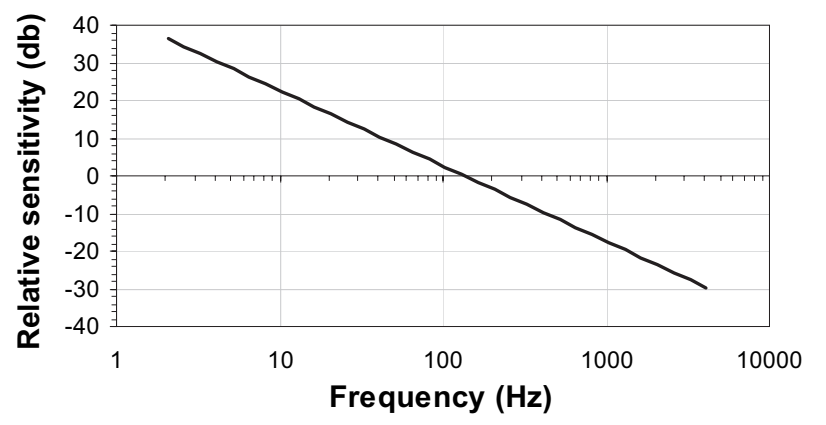

FIG. 2 Response of geophone vs. accelerometer with respect to frequency

$$
\text { Relative Sensitivity }(d B)=20 \log (U v / U a)
$$

where $U v$ and $U a$ are the voltages registered by a geophone and an accelerometer, respectively. Let us consider a $15 \mathrm{~Hz}$ geophone with a sensitivity of $0.43 \mathrm{~V} / \mathrm{cm} / \mathrm{s}$ (G1-15) and a $0.5 \mathrm{~V} / \mathrm{g}$. accelerometer (A1-0.5). Assuming a stress drop of $1 \mathrm{MPa}$, the moment magnitude $\left(M_{w}\right)$ can be related to the corner or characteristic freqency $(f)$ by:

$$
M_{w}=(2.11-\log (f)] / 0.5
$$

The results are presented in Figure 3.

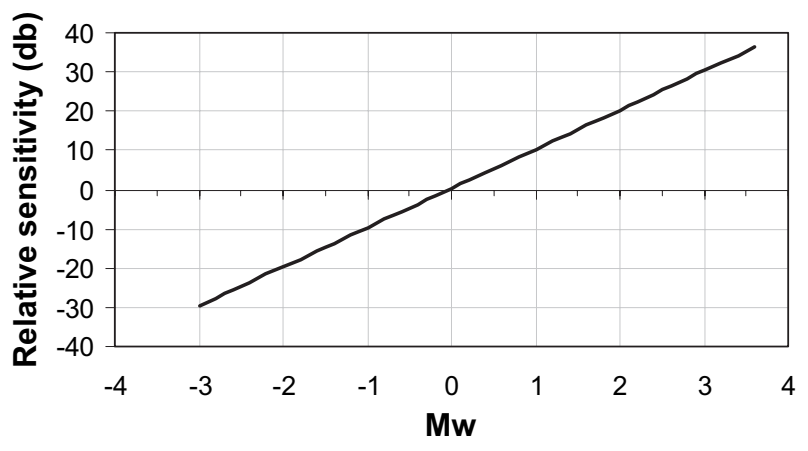

FIG. 3 Response of geophone vs. accelerometer with respect to moment magnitude

From Figures 2 and 3 it is apparent that geophones are better suited to measuring lower frequencies $(f<100 \mathrm{~Hz})$ or larger magnitudes $\left(M_{w}>0\right)$. Accelerometers are more appropriate for measuring higher frequencies $(f>100 \mathrm{~Hz})$ or smaller magnitudes $\left(M_{w}<0\right)$. In most Canadian mines where there are few large events, accelerometers are used to measure microseismicity in the $M_{w}$ range -2 to 0 . To cover the less frequent larger events, one or more geophones are installed on or near surface, approximately $1 \mathrm{~km}$ from the mining activity.

The measured effective response of the G1-15 is shown in Figure 4 for the reference $0.1 \mathrm{~V}$ corresponding to a vibration of $0.2 \mathrm{~g}$ recorded by the A1-0.5. The response of the $30 \mathrm{~V} / \mathrm{g}$ most commonly used accelerometer (A1-30) is also shown.

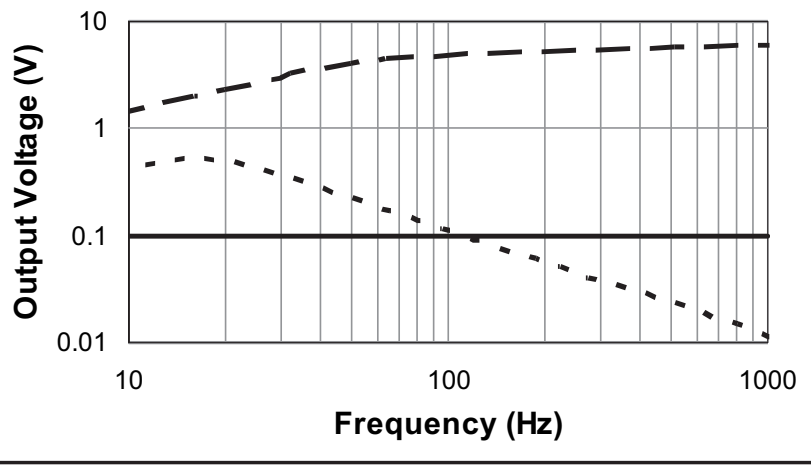

FIG. 4 The response of a $15-\mathrm{Hz}$ geophone (dotted line) to a 0.2 $\mathrm{g}$ vibration on a $0.5 \mathrm{~V} / \mathrm{g}$ accelerometer (thick solid line); dashed line is the response of a $30 \mathrm{~V} / \mathrm{g}$ accelerometer

\subsection{Centralized Acquisition}

Measuring microseismicity was accomplished by using a high sampling rate $(20 \mathrm{kHz})$ and tight array geometries with inter-sensor distances typically less than $100 \mathrm{~m}$. Timing accuracy was critical so a centralized recording architecture was adopted allowing simultaneous sampling of all sensors with microsecond accuracy and the dynamic range of the system was increased to over $90 \mathrm{~dB}$ using 16-bit analogue to digital conversion.

This centralized acquisition system (Figure 5) offered a simple robust design with one power point that made it very easy to troubleshoot wiring problems and bad sensors. Computer and electronic equipment resided in the engineering office on surface and only the inexpensive (disposable) sensors were subject to the harsh mining conditions. However, as mines developed at depth the seismic systems were expanded to over 90 channels. These large systems were often monitoring several different mining 
zones within the same mine. Problems developed as seismic events from different mining horizons began to interfere with one another. To overcome these difficulties a multiarray technology was developed, allowing larger systems to be divided up into smaller sub-systems or zones. Multiple zones are triggered and processed independently but still allow for some sensor sharing along zone boundaries. Also, the cost to extend and maintain the twisted pair copper cable infrastructure on these large systems became prohibitive. Excessively long cable runs were susceptible to electrical interference, degrading the signal quality and system performance.

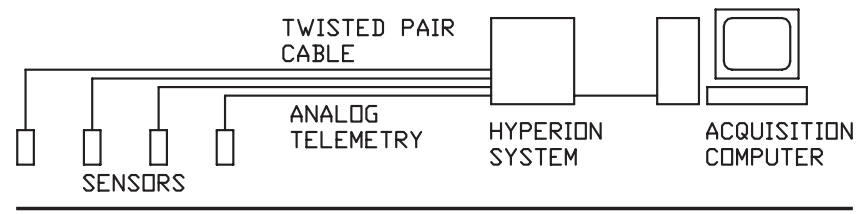

FIG. 5 Centralized configuration

\subsection{Fibre Optic Communication}

Fibre optic cable, while no more expensive than copper to install, has a much higher bandwidth. It allows for up to 64 channels of seismic data sampled at $20 \mathrm{kHz}$ to be multiplexed onto 2 fibre cores (Tx, Rx). Data is transmitted digitally with no signal degradation and is not susceptible to electrical interference. The disadvantage of fibre is that it requires special equipment and training to install and is more difficult to repair than copper. Thus, fibre cables are usually limited to the shaft area or main access ways.

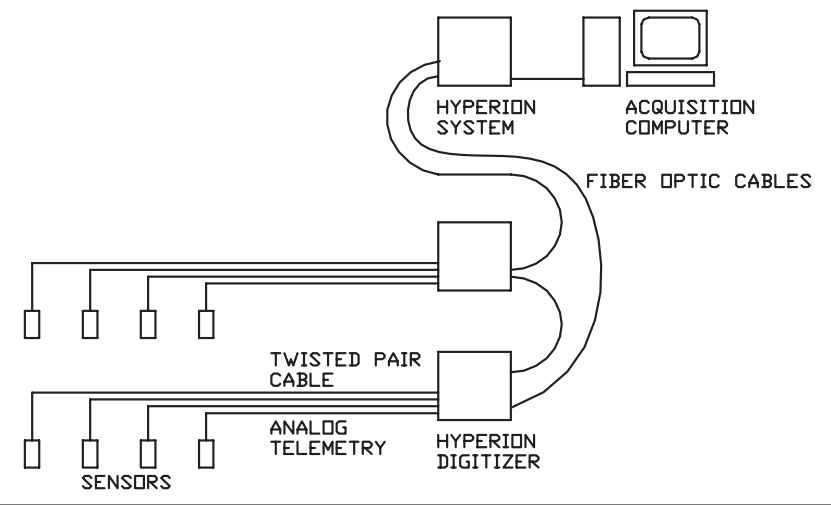

FIG. 6 Daisy chain fibre optic communication

As shown in Figure 6, twisted pair copper cable is used to transmit the analogue base band signal from the sensor to the shaft area. Fibre optic transceivers are installed on multiple mine levels to digitize the signal from each sensor on that level and multiplex the data onto a fibre optic trunk cable running up the shaft. The continuous data signal from each sensor is monitored on an acquisition computer in the engineering office on surface. Complex triggering logic monitors the signal amplitude on each channel to automatically determine when an event has occurred.

\section{SYSTEM OPERATIONS}

Automatic diagnostic tools assimilate data from the hardware and software components (e.g., sensor voltage levels, fibre synchronization status, trigger and arrival time data) and process this information to help determine problematic sensors or hardware failures. This diagnostic status information is used to troubleshoot, optimize system performance, and notify key personnel in the event of a critical failure, allowing for corrective or preventive action to be taken in a timely manner.

A 3D visualisation tool is the main user interface to most systems, primarily used to display location and magnitude information with respect to mine workings. Having the full waveform data from each station allows trained operators to manually check the automatic picks and reprocess the data to obtain the best event locations possible. A read-only version of the visualisation software is used by security and management to view the system information from anywhere on the network. A suite of processing and reporting tools is used to help determine the blast re-entry protocol and identify active faults or regions of instability.

\subsection{Accurate Event Locations}

Newmont's Golden Giant mine located in Marathon, Ontario, is situated between the Williams and David Bell mines (Figure 7). Since ground problems were anticipated, a centralized seismic system was installed early in mine life.

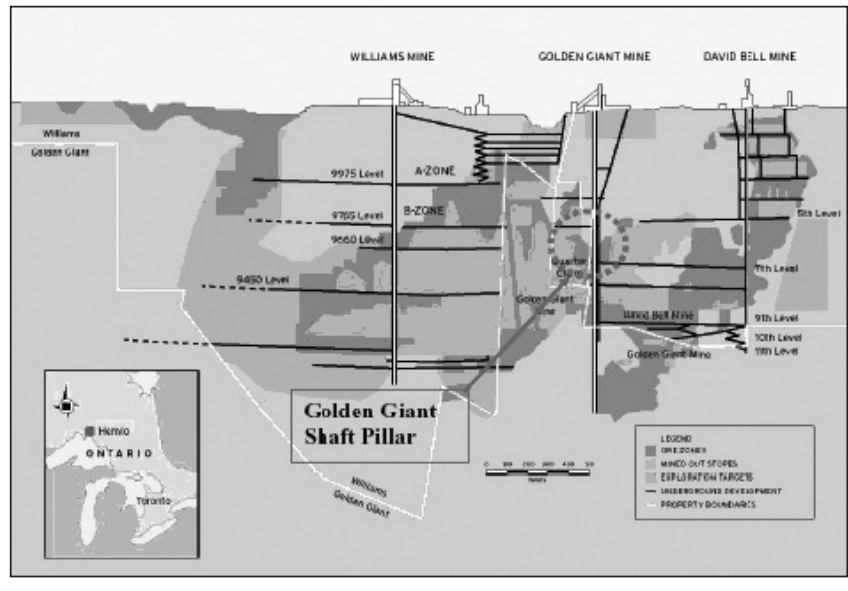

FIG. 7 Golden Giant mine

In 1990 blasting to within $30 \mathrm{~m}$ of shaft caused minor loose ground to be shaken off the shaft walls, and in 1995 relatively intense microseismic activity led to visible deterioration of 4600 shaft station and surrounding development, resulting in the temporary closure of the mine. Following re-support of the shaft there were increasing signs of stress. The shaft pillar contained high-grade ore and the original plan was to mine below 4600L and extract the shaft pillar as the final mining block. However, economics required the shaft pillar and deep ore to be mined simultaneously. A de-stress slot concept was proposed which would be $90 \mathrm{~m}$ high by $50 \mathrm{~m}$ wide (3 stopes) positioned north of the shaft (McMullan et al., 2004). This called for mining both ore and waste to within $8 \mathrm{~m}$ of the shaft (inside the previously damaged area). The seismic system was expanded via fibre optics to an 88 channel system with 40 channels monitoring mine-wide, 16 channels focused on the deep zone and a specialized 32 channel dense array around the shaft pillar (Figure 8). Location accuracy better than $4 \mathrm{~m}$ was required within the dense shaft pillar array. 


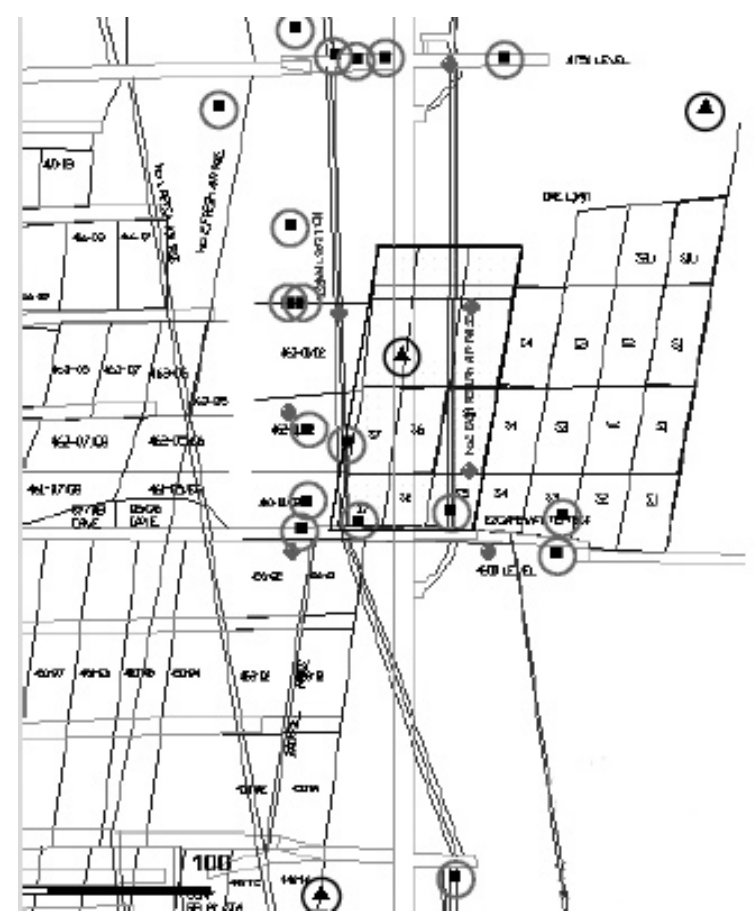

FIG. 8 Shaft pillar array. Each circle corresponds to the location of a seismic sensor. The square or triangle inside the circle denotes uniaxial or triaxial accelerometer, respectively

From April 2002 to June 2003 more than 15,000 events were processed, with the results summarized in Figure 9 (Mercer, 2004). Monitoring the entire shaft pillar in real-time, the seismic system confirmed that both hangingwall and footwall of the slot were de-stressed, and provided timely insight into relative loading condition changes within this pillar.

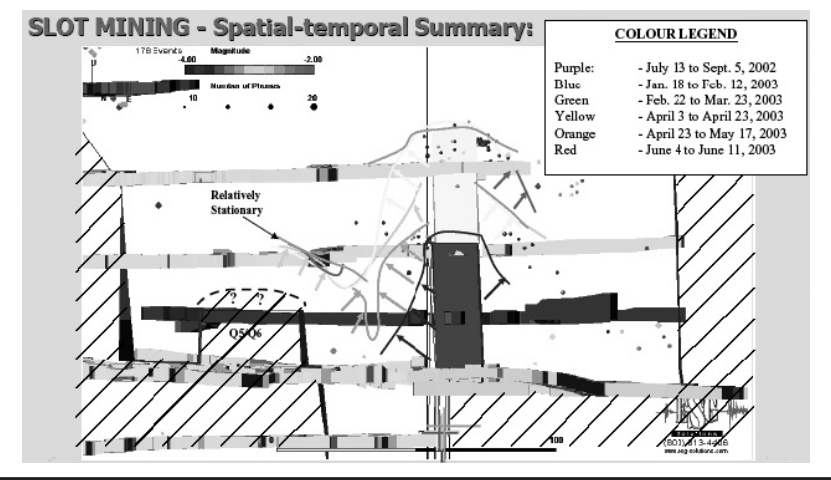

FIG. 9 Slot mining spatial-temporal summary

\subsection{Large Event / Blast Re-entry Protocol}

Inco's Creighton mine located in Sudbury, Ontario, has been mining since 1901. An 80-channel daisy chained fibre optic seismic system monitors from 3800 level down to 7200 level. The mine employs seismic information to enhance their large event / blast re-entry protocol. To simplify the decision making, five parameters were chosen which can be reliably estimated by the system in real-time and for which a reasonable background estimate can be obtained.

(1) Seismic work, derived from the seismic moment $\left(M_{0}\right)$ and plotted as a cumulative parameter (Figure 10), is a measure of the rate of seismic event generation. Non-linear slopes indicate unstable conditions (critical phenomena), such as rockburst sequences, whereas linear slopes indicate return to background conditions (non-critical state).

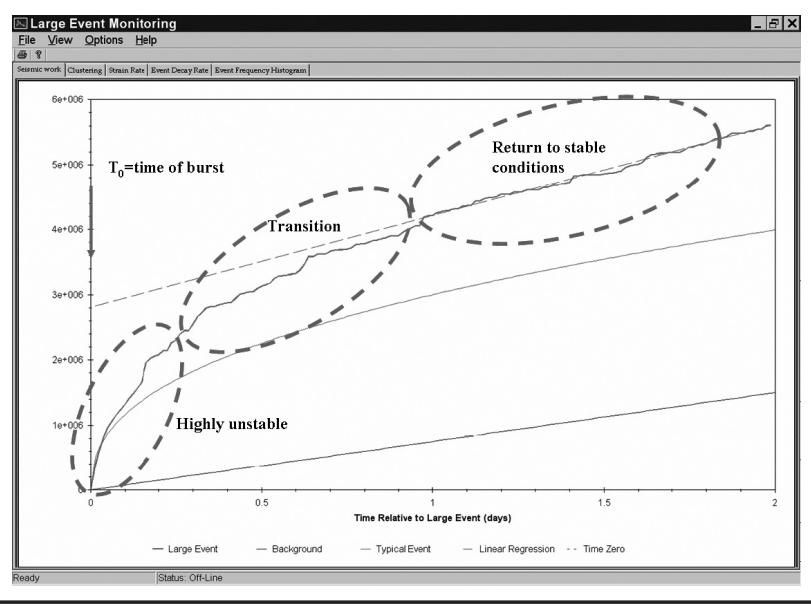

FIG. 10 Seismic work

(2) Clustering measures event dispersion throughout the mine and indicates if seismic events are grouping together. (3) Seismic strain rate represents the deformation change, in a particular region, resulting from the occurrence of a sequence of microseismic events. For example, many events occurring in a small volume over a short period of time will yield a high seismic strain rate. (4) Event decay rate indicates the frequency of seismic events. According to Omori's law, this rate is inversely proportional to the time elapsed since the occurrence of the large event. (5) Histograms of activity by level assist in identifying where clustering occurs relative to the location of the large event and if activity migrates to other areas.

When a large event occurs the location is checked with the seismic system and personnel evacuated from the area. The operator sets the time zero from which the numbers are calculated and then monitors the online parameters. If these parameters show the expected decay and return to normal background levels, then it may be safe to return underground; otherwise, additional areas may have to be evacuated.

\subsection{Event Mechanism Solutions}

Falconbridge's Kidd Creek mine in Timmins, Ontario, was opened in the mid 1960s. The copper-zinc rich plug-shaped orebody, round across, has a significant influence on the size and rate of seismicity, ensuring a soft load transfer from the host rock to the pillars as the ore is extracted (Brummer et al., 1996). Similar to Creighton, mining here is scheduled to take place over the next years at $3 \mathrm{~km}$ underground, making them the deepest metal base, bulk operations in the world.

Kidd Creek operates a 64-channel system for the monitoring of the shallow \#1 and \#2 mines, from below the open pit down to 4600 level, and another 64-channel system to cover \#3 mine that extends to 6800 level. Finally, 'D' mine will employ in the first phase a 48-channel system to monitor down to the 8800 level (Figure 11).

Event mechanism solutions are calculated on site for events with magnitudes larger than -1 that trigger at least 5 of the 12 triaxial accelerometers. Rapid evaluation employs a moment tensor algorithm that calculates low frequency amplitude displacements in the time domain, with the operator confirming the identification of the $\mathrm{P}, \mathrm{SV}$ and $\mathrm{SH}$ first arrival polarities (Trifu et al., 2000; Trifu and Shumila, 2002).

Event mechanism solutions at Kidd represent additional information used to investigate various aspects related to ground control. For example, the identification of non-shear failures in a sill pillar between \#2 and \#3 mines led to the conclusion that the pillar has not been damaged and can continue to be used for efficient hauling. In a different example, the identification of shear mechanism solutions in 


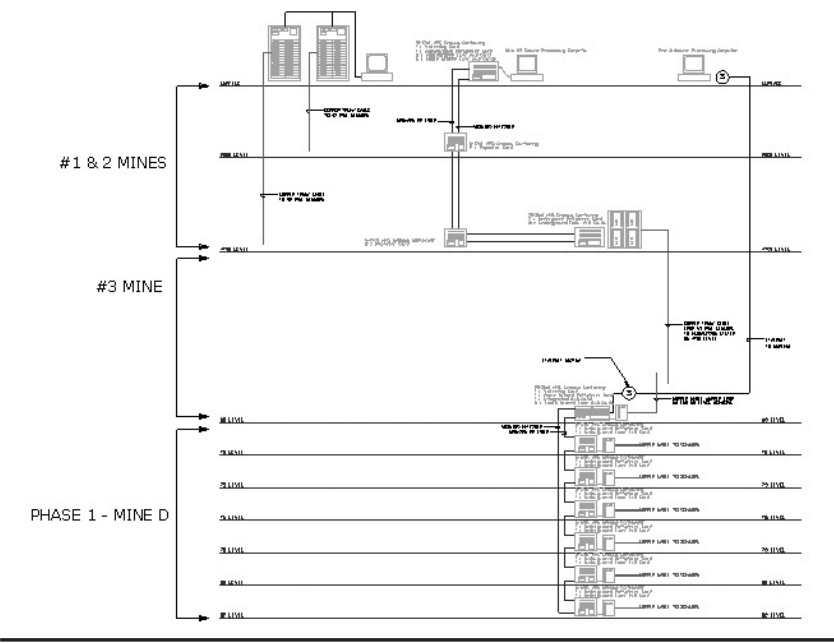

FIG. 11 Seismic system configuration at Kidd Creek mine

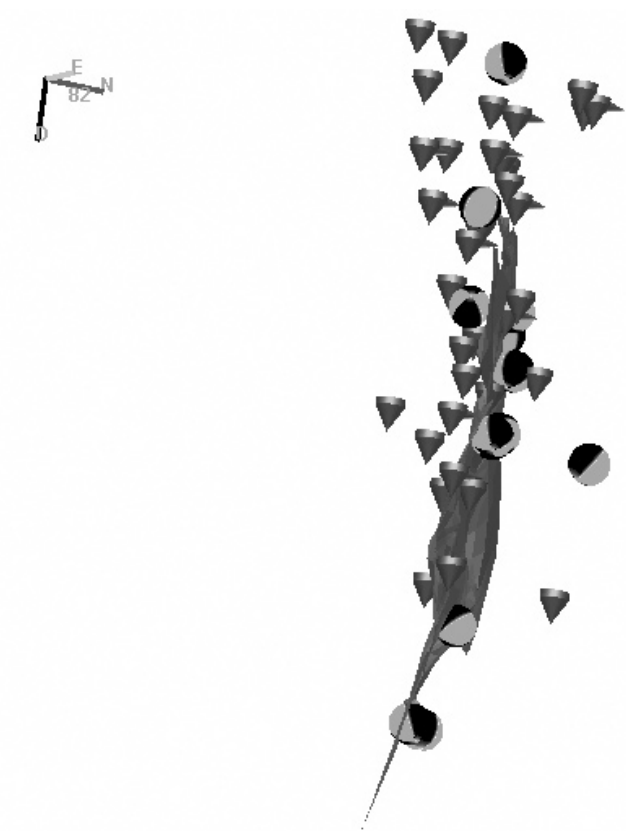

FIG. 12 Event mechanism solutions for event associated with the North F fault at Kidd Creek mine

agreement with the orientation of larger magnitude seismicity in the proximity of the North F fault was indicative that this structure was active below 6800 level, information used in the design of a large mechanical shop in mine ' $\mathrm{D}$ ' (Figure 12).

Although these tight microseismic arrays offer very accurate event locations, they can be susceptible to clipping on larger events or blasts. To properly record the larger seismic activity, low frequency digital recorders with $4.5 \mathrm{~Hz}$ geophones are typically located on or near surface, about $1 \mathrm{~km}$ from the mining activity. Seismic data is acquired and processed on a standard PC, which can save event location and magnitude information with data from the underground microseismic system through the local area network (LAN). Almost all Canadian mines use one or more of these seismographs to supplement the underground microseismic array.

\section{TRENDS IN SEISMIC MONITORING}

With the expansion of underground mines and open pits came an increase in demand for small regional surface networks that could monitor seismicity across several mining camps. Many of these remote sites require power, wireless connectivity, and persistent local data storage. For these applications, a distributed network of low power 24-bit web enabled seismic recorders is deployed using solar power, industry standard spread spectrum wireless Ethernet, and GPS time synchronization (Figure 13). Continuous (or event) data is transmitted back to a central site where a network acquisition program merges the data streams from multiple remote sites and processes the information exactly as with underground networks.

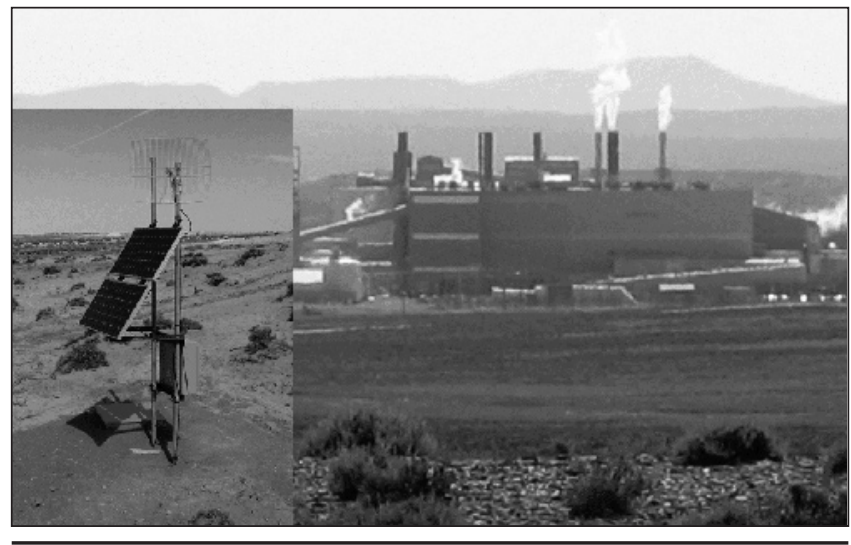

FIG. 13 Remote seismic surface array

Most Canadian mines now extend Ethernet LAN connectivity underground. This communication backbone can be used to support a wide variety of network, monitoring, and control systems. Recent advances in high-speed connectivity using standard telephone cable offer some new opportunities for underground Ethernet communication. DSL (Digital Subscriber Line) is a technology for bringing high-bandwidth information to homes and small businesses over ordinary copper telephone lines (Table 1).

TABLE 1 Typical DSL capabilities

\begin{tabular}{lll}
\hline & Symmetric & Asymmetric \\
\hline Max. Distance & $1.4 \mathrm{~km} @ 6 \mathrm{Mbps}$ & $2 \mathrm{~km} @ 4 / 1.5 \mathrm{Mbps}$ \\
Max. Speed & $16 \mathrm{Mbps} @ 1 \mathrm{~km}$ & 16/2Mbps @ 1.5 km \\
\hline
\end{tabular}

This technology is now being used to extend the underground Ethernet backbone to support a distributed network of underground microseismic recorders. In this way, a mine has one affordable communication infrastructure to install, maintain and support, which is flexible, robust, and secure. It will allow the seamless integration of seismic data from both underground and surface stations.

\section{CONCLUSIONS}

The monitoring of mine seismicity has been carried out in Canada for more than two decades. Throughout this period, the Canadian mining industry has benefited from a long series of technological and scientific advancements, which contributed to the development of hardware and software solutions that ensure highly sensitive event detection, increased location accuracy, and fully automatic operation.

Full waveform event based microseismic systems are commonly employed. Using 16-bit resolution, $20 \mathrm{kHz}$ sampling, and a combination of uniaxial and triaxial accelerometers, such systems monitor mine seismicity ranging in moment magnitude between approximately -2 and 0 , with location accuracy ranging from meters to a few tens of meters. Larger magnitude events are generally monitored using surface geophones. From the largest underground block caving operation to a small narrow-vein gold operation, 
tens of Canadian mines operate seismic networks, using monitoring results in their assessment of ground conditions, mine design, personnel and mine safety.

The expansion of Ethernet LAN underground has created support communication conditions for continuous microseismic data acquisition from a distributed network of microseismic recorders with 24-bit resolution, operating at sampling frequencies as high as $10 \mathrm{kHz}$. The same web enabled, low power recorders can be equally installed on surface, where they can operate using solar power, GPS time synchronization, and radio Ethernet communication.

\section{REFERENCES}

Blake, W. (1982) Microseismic applications for Mining. A Practical guide, Report J0215002, US Bureau of Mines.

Hedley, D.G.F. (1992) Rockburst handbook for Ontario hard-rock mines, CANMET Special Report SP92-1E, Minister of Supply and Services Canada, Ottawa.

McMullan, J., Bawden, W.F. and Mercer, R. (2004) Excavation of a shaft destress slot at the Newmont Canada Golden Giant Mine, in Proceedings 6th North American Rock mechanics Symposium, D. P. Yale, S.M. Willson, and A.S. Abou-Sayed (Editors), Houston, Texas, June 5-10, 2004

Mercer, R. (2004) The collection and interpretation of seismic data generated during the excavation of a shaft de-stress slot, Proceedings of the Monitoring Mining Induced Seismicity Workshop, September 21-23, 2004, Kingston, Ontario, Section 6, 62 p.

Trifu, C-I. and Shumila, V. (2002) Reliability of seismic moment tensor inversions for induced microseismicity at Kidd mine, Ontario, PAGEOPH 159, pp. 145-164.

Trifu, C-I. and Urbancic, T.I. (1997) Characterization of rockmass behaviour using mining induced seismicity, CIM Bulletin 90 (1013), pp. 62-68.

Trifu, C-I., Angus, D. and Shumila, V. (2000) A fast evaluation of the seismic moment tensor for induced seismicity, Bull. Seismol. Soc. Am. 90, pp. 1521-1527. 